\title{
2 Schizophrenie und akute vorübergehende Psychosen
}

\author{
Thomas Stompe und Wielant Machleidt
}

Schizophrenie war in den letzten 50 Jahren häufig ein zentraler Untersuchungsgegenstand der kulturvergleichenden Psychiatrie. Daneben wurden vor allem in ethnopsychiatrisch orientierten Arbeiten akute, psychotische Zustandsbilder beschrieben, die im Querschnitt schizophrenen Erkrankungen ähnlich sehen, sich aber durch einen günstigen Verlauf mit kompletten Remissionen auszeichnen. Im Folgenden sollen beide Formen vorgestellt werden, wobei der Schwerpunkt allerdings auf den Darstellungen der Schizophrenie liegt.

\subsection{Schizophrenie}

Schizophrenie ist eine zumeist schubhaft oder chronisch verlaufende psychotische Erkrankung mit einer durchschnittlichen Lebenszeitprävalenz von o,51\%. Aufgrund der häufig ungünstigen Verläufe übersteigt die volkswirtschaftliche Bedeutung die realen Prävalenzen deutlich. Seit dem 19. Jahrhundert finden sich gut identifizierbare Beschreibungen schizophrener Krankheitsbilder. Der Terminus selbst wurde von Eugen Bleuler (1911) in die psychiatrische Fachliteratur eingeführt und löste rasch den von Emil Kraepelin geprägten Begriff der Dementia praecox ab. Die genaue Definition, der Umfang und die Binnendifferenzierung der Schizophrenie blieben während des gesamten 20. Jahrhunderts umstritten. Auch die Kriterien der inzwischen allgemein gebräuchlichen Klassifikationssysteme der World Health Organization (ICD10) und der American Psychiatric Association (DSM-IV) unterscheiden sich in wichtigen Details (s. Tab. 3). 
Tab. 3 Schizophrenie in der ICD-10 (WH0 1993) und im DSM-IV (APA 1996)

\begin{tabular}{|c|c|c|}
\hline & ICD-10 & DSM-IV \\
\hline Symptomatik & $\begin{array}{l}\text { 1. Gedankenlautwerden, -eingebung, -entzug, -aus- } \\
\text { breitung } \\
\text { 2. Kontroll-, Beeinflussungswahn, Gefühl } \\
\text { des Gemachten, Wahnwahrnehmungen } \\
\text { 3. kommentierende-, dialogische Stimmen } \\
\text { 4. anhaltender, kulturell unangemessener oder } \\
\text { völlig unrealistischer Wahn } \\
\text { 5. anhaltende Halluzinationen jeder Sinnes- } \\
\text { modalität } \\
\text { 6. Gedankenabreißen, Einschiebungen in den } \\
\text { Gedankenfluss, Zerfahrenheit, Danebenreden, } \\
\text { Neologismen } \\
\text { 7. katatone Symptome wie Erregung, Haltungs- } \\
\text { stereotypien, wächserne Biegsamkeit, } \\
\text { Negativismus, Mutismus, Stupor } \\
\text { 8. negative Symptome wie Apathie, Sprach- } \\
\text { verarmung, verflachter oder inadäquater Affekt }\end{array}$ & $\begin{array}{l}\text { 1. Wahn } \\
\text { 2. Halluzinationen } \\
\text { 3. desorganisierte Sprechweise } \\
\text { 4. desorganisiertes oder } \\
\text { katatones Verhalten } \\
\text { 5. negative Symptome } \\
\text { 6. mindestens } 2 \text { von } 5\end{array}$ \\
\hline Folgen & - & $\begin{array}{l}\text { soziale/berufliche Leistungs- } \\
\text { einbußen }\end{array}$ \\
\hline Zeitkriterium & $\begin{array}{l}\text { mindestens ein eindeutiges Symptom von 1-4 } \\
\text { oder mindestens zwei von 5-8 fast ständig } \\
\text { während eines Monats }\end{array}$ & $\begin{array}{l}\text { Zeichen des Störungsbildes } \\
\text { halten mindestens } 6 \text { Monate an }\end{array}$ \\
\hline
\end{tabular}

Beide Klassifikationssysteme greifen dabei auf deutschsprachige Klassiker der psychopathologischen Schizophrenieforschung zurück. Die WHO (1993) verwendet für die Beschreibung des Symptomatik vorwiegend die Schneider'schen Symptome ersten Ranges (Schneider 1952), die APA (1996) hingegen die akzessorischen Symptome Eugen Bleulers (1911). Das Zeitkriterium der WHO verweist auf Bleulers Annahmen über die Existenz günstiger schizophrener Verläufe, während die APA sich eher den pessimistischen Annahmen Kraepelins anschließt.

Die Ätiologie der Schizophrenie ist weitgehend ungeklärt. Man geht von einer komplexen Genese aus, in der biologische, biographische und soziokulturelle Faktoren interagieren. Wissenschaftliche Untersuchungen in fremden Kulturen und die Begegnung mit psychisch kranken Migranten hoben deutlich die große Rolle der kulturellen Rahmenbedingungen hervor. Es gilt daher in Folge, die Bedeutung der kulturvergleichenden Psychiatrie für die Schizophrenieforschung der Gegenwart herauszustreichen.

\subsubsection{Geschichte der kulturvergleichenden Schizophrenieforschung}

Emil Kraepelin war im deutschen Sprachraum der Erste, der die Bedeutung von kulturvergleichenden Untersuchungen für die Psychiatrie erkannte: 
„Bisher haben sich alle vergleichend psychiatrischen Untersuchungen auf die Gruppenbildung innerhalb eines Volkes beschränkt. Wir sind uns durchaus im Unklaren darüber, ob und in welchen Punkten sich die psychische Morbidität der Franzosen, Engländer, Italiener von der unsrigen unterscheidet. Ein irgendwie zuverlässiger Vergleich ist natürlich auch erst dann möglich, wenn wir nicht allein Zustandsbilder, sondern wirkliche Krankheitsformen auseinander zu halten vermögen, und er kann wegen der Verschiedenheit der klinischen Auffassungen für absehbare Zeit nur von ein und demselben Beobachter durchgeführt werden. Aus diesem Grunde sind die Angaben über die psychischen Erkrankungen fremder Menschenrassen nur in einigen wenigen Punkten verwertbar, obgleich an sich gerade die Größe der hier zu erwartenden Unterschiede den Vergleich besonders fruchtbar und zuverlässig machen sollte." (Kraepelin 1904a)

Kraepelin entwarf damit ein für diese Zeit richtungweisendes theoretisches Programm für ein neues psychiatrisches Forschungsfeld (Bendick 1989; Boroffka 1989). Er war aber auch erste Psychiater aus dem deutschsprachigen Raum, der systematisch eine außereuropäische Krankenhauspopulation untersuchte (Kraepelin 1904b). Mit dem Erkenntnisinteresse ausgestattet, das ubiquitäre Vorkommen der von ihm postulierten Dichotomie der endogenen Psychosen in Manisch-depressives Irresein und der Dementia praecox nachzuweisen, besuchte Kraepelin während einer Weltreise ein holländisches Kolonialspital in Java, wo er mit Unterstützung von einheimischen Dolmetschern mehr als 100 stationär aufgenommene Patienten persönlich explorierte. Er fand heraus, dass Fälle von Dementia praecox unter den Einheimischen häufiger vorkamen als unter den europäischen Kolonialherren. Im Vergleich zu den Patienten mit Dementia praecox, die Kraepelin in Deutschland untersucht hatte, traten katatone Störungen bei den Javanesen nur gelegentlich auf, auch akustische Halluzinationen waren deutlich seltener als in Europa, zusammenhängende Wahnbildungen fanden sich nur angedeutet oder fehlten überhaupt vollständig. Initiale depressive Zustände ebenso wie heftige Erregungszustände gab es nur selten, auch schwere Residualverfassungen („Verblödung“) wurden in der Regel nicht erreicht. Die Endzustände boten meist „das Bild einer gewissen faseligen Verworrenheit und Unklarheit ohne äußerlich besonders auffallende Begleiterscheinungen“ (Kraepelin 1904a, S. 435). Kraepelin zog daraus den Schluss, dass kein einziges der in Deutschland geläufigen Symptome der Dementia praecox fehlte, deren Ausprägung insgesamt aber weniger deutlich war. Kraepelins sozial-darwinistisches Erklärungsmodell für die vorgefundenen Unterschiede ist ein Spiegel des kolonial-eurozentristischen Denkens des imperialen Zeitalters:

„Insbesondere könnte die Geringfügigkeit der Wahnbildungen mit dem niedrigen Stand der geistigen Entwicklung und die Seltenheit der Gehörstäuschungen mit dem Umstande in Beziehung stehen, dass die Sprache für das mehrin Sinnesvorstellungen sich bewegende Denken nicht die Bedeutung hat, wie bei uns“" Kraepelin 1904a, S. 436).

Aus der Perspektive der kulturvergleichenden Schizophrenieforschung war die Periode nach den Kraepelins Aufzeichnungen bis 1955 durch einen relativen Stillstand gekennzeichnet. In den Kolonien der europäischen Nationen beschränkten man sich auf die Auswertung von Statistiken psychiatrischer 
Krankenhäuser, die allerdings mehr das Inanspruchnahmeverhalten als reale Prävalenzen abbildeten (s. Abb. 5).

Die Unterschiede sind so deutlich, dass populationsbiologische Mechanismen oder Umweltfaktoren wie Infektionserkrankungen oder Sozialisationsbedingungen die gesamte Spannweite dieser Krankenhausprävalenzen nicht erklären können. Vielmehr bilden sich hier vermutlich auch Zugangsbarrieren zur westlichen medizinischen Behandlung und/oder die Dominanz traditioneller Medizinsysteme ab.

Daneben gab es vereinzelt ethnopsychiatrische Untersuchungen wie die detaillierten Aufzeichnungen von Georg Devereux über die therapeutischen Gespräche mit einem schizophrenen Mann vom Stamme der Sioux (Devereux 1961, 1985). Weitere psychiatrische Feldforschungen in traditionellen Stammesgesellschaften aus dieser Zeit belegten, dass Schizophrenie in einer weiten Definition in allen untersuchten Gemeinschaften vorkommt. Beispiele sind die Untersuchungen von Salisbury bei den Siane im Hochland von Neuguinea, von Cawte über die Kalamuru- und Walbiri-Aborigines in Australien, von Aall-jilek über die Wagoporo in Tanzania, von Teicher über die Inuits in der nördlichen HudsonbayRegion und von Spiro über die Melanesier von Ifaluk (Pfeiffer 1994). Zwar erkannten in dieser Zeit die meisten Autoren an, dass Schizophrenie ein ubiquitär vorkommendes Krankheitsbild ist, dem kulturanthropologischen Ansatz folgend wurden jedoch mehr die Unterschiede als die Ähnlichkeiten hervorgehoben. So wurde bereits in den 1950er-Jahren von afrikanischen Nervenärzten wie Lambo (1957) die Übertragbarkeit westlicher Diagnosekonzepte auf Stammesgesellschaften kritisch hinterfragt. Hervorgehoben wurde dabei, dass die Psychosen in afrikanischen Gemeinschaften nur selten einen chronischen Verlauf nehmen und fast nie dem Bild einer schizophrenen Prozesspsychose entsprechen.

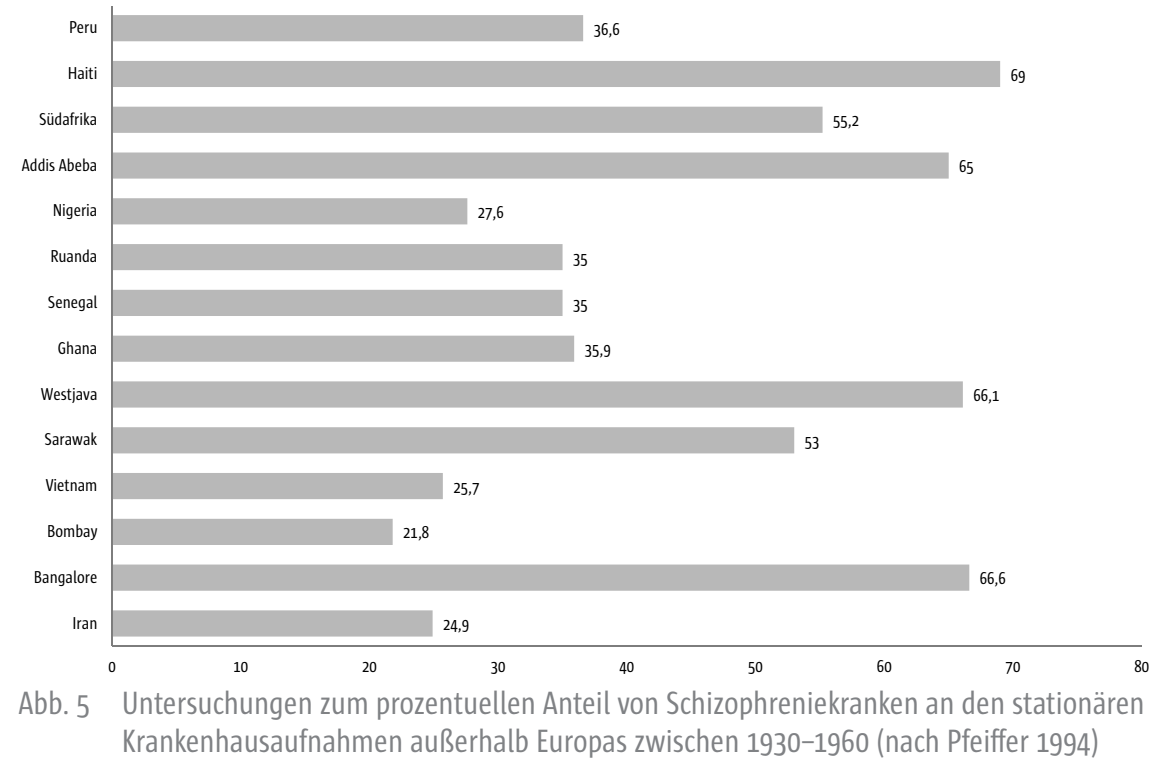


Entscheidende neue Impulse für die kulturvergleichende Schizophrenieforschung kamen nach dem 2. Weltkrieg aus Kanada. 1955 gründeten Eric Wittkower und Jack Fried in Montreal die Sektion für transkulturell-psychiatrische Studien als Kooperation zwischen der psychiatrischen Abteilung und dem anthropologischen Institut der McGill Universität. In dieser Arbeitsgruppe war es Murphy, der sich am intensivsten mit transkulturellen Aspekten der Schizophrenie auseinandersetzte. Um einen ersten umfassenden Überblick zur kulturellen Verteilung schizophrener Symptome zu gewinnen, schickte er 1960 einen Fragebogen an 40 Psychiater in 27 Ländern aus. Erhoben wurde darin, in welcher Weise sich die Patienten aus der Kultur des behandelnden Arztes von den Kranken aus anderen Kulturen unterscheiden. Viele Ergebnisse der Studien dieser Arbeitsgruppe sind zu einem festen Bestandteil des transkulturell-psychiatrischen Diskurses über die Schizophrenie geworden (s. Tab. 4) und wurden in späteren, methodisch aufwändigeren, dafür aller-

\section{Tab. 4 Vorkommen schizophrener Symptome nach Murphy (1963)}

\begin{tabular}{|c|c|}
\hline schizophrene Symptome und Syndrome & soziokulturelle Korrelate \\
\hline \multirow[t]{2}{*}{ paranoider Typus } & am häufigsten in der urbanen Mittelklasse \\
\hline & am seltensten in der Landbevölkerung \\
\hline katatoner Typus & am seltensten bei Euro-Amerikanern \\
\hline hebephrener Typus & am häufigsten bei Japanern \\
\hline simplex Typus & am häufigsten bei Asiaten \\
\hline \multirow[t]{2}{*}{ visuelle Halluzinationen } & am häufigsten in Afrika und im Nahen Osten \\
\hline & am seltensten bei städtischen Euro-Amerikanern \\
\hline taktile Halluzinationen & am häufigsten in Afrika und im Nahen Osten \\
\hline Größenwahn & am häufigsten in der Landbevölkerung \\
\hline Weltuntergangswahn & am häufigsten bei Christen \\
\hline religiöser Wahn & am häufigsten bei Christen \\
\hline Eifersuchtswahn & am häufigsten bei Asiaten \\
\hline Depersonalisation & am häufigsten in der Landbevölkerung \\
\hline \multirow[t]{2}{*}{ Affektverflachung } & am häufigsten bei Japanern \\
\hline & häufig in Südamerika \\
\hline sozialer Rückzug & am häufigsten bei Japanern \\
\hline Negativismus & am häufigsten in Indien und Südamerika \\
\hline \multirow[t]{2}{*}{ Erregungszustände } & am häufigsten in Afrika und Südamerika \\
\hline & am seltensten in angelsächsischen Ländern \\
\hline \multirow[t]{2}{*}{ Stereotypien } & am häufigsten in Hinterindien \\
\hline & am seltensten bei Euro-Amerikanern \\
\hline katatone Starre & am häufigsten in Hinterindien \\
\hline Suizidalität & am häufigsten bei Japanern \\
\hline Gefühlsausbrüche & am häufigsten bei Christen \\
\hline
\end{tabular}


dings weniger breit angelegten Untersuchungen wiederholt bestätigt (Murphy et al. 1963; Murphy u. Raman 1971; Wittkower et al. 1960).

Mit der International Pilot Study of Schizophrenia (IPSS) fand die transkulturelle Psychiatrie endgültig Anschluss an die strengen methodischen Maßstäbe der Gegenwartspsychiatrie. Die Planung dieser klinischen und epidemiologischen kulturvergleichenden Untersuchungen erfolgte durch die World Health Organization in den späten 196oer-Jahren (WHO 1973). Die IPSS umfasste neun Zentren aus Afrika (Nigeria), Asien (Taiwan, Indien), Europa (Großbritannien, Dänemark, Tschechoslowakei, UDSSR) und Amerika (USA, Kolumbien) (Sartorius et al. 1972). Nach zwei Jahren durchliefen 82\% der ursprünglichen Kohorte die komplette neuerliche Untersuchung, nach fünf Jahren immerhin noch 75\% (Sartorius et al. 1977, 1978, 1996; Jablensky et al. 1992). Trotz zum Teil berechtigter Kritik setzte die International Pilot Study of Schizophrenia Maßstäbe für alle zukünftigen kulturvergleichenden Untersuchungen auf dem Feld der epidemiologischen Psychiatrie. Die IPSS war der Auftakt für weitere große Schizophreniestudien der WHO, wie die zehn Länder umfassende „Determinants of Outcome of Severe Mental Disorders“ (Edgerton u. Cohen 1994) oder zu der „International Study of Schizophrenia“(Hopper et al. 20o1). Auch außerhalb der WHO-Studien etablierten sich inzwischen länderübergreifende Kooperationen wie die sieben Länder umfassende „International Study on Psychotic Symptoms“ (ISPS) (Stompe u. Friedmann 2007).

\subsubsection{Epidemiologie}

Wie bereits erwähnt, beruhte die Epidemiologie der Schizophrenie in außereuropäischen Kulturen in den ersten Jahrzehnten des 20. Jahrhunderts im Wesentlichen auf Spitalsstatistiken. Seit den 197oer-Jahren wandelte sich das Bild unter dem Eindruck der Ergebnisse der großen kulturvergleichenden Untersuchungen der WHO. Nunmehr ging man von einer nahezu gleichmäßigen Verteilung der Schizophrenie in allen Teilen der Welt aus, ein Resultat, das allerdings ebenfalls erhebliche Fragen aufwarf. In der Zwischenzeit hat sich eine differenziertere, realitätsgerechtere Sichtweise etabliert, die sowohl Ähnlichkeiten als auch Unterschiede in der Verteilung schizophrener Erkrankungen interpretieren kann.

\section{Inzidenz}

Ende der 7oer-Jahre wurde von der WHO eine internationale Untersuchung in Nachfolge der IPSS initiiert, die Studie über Determinanten schwerer psychischer Störungen (WHO Collaborative Study on the Determinants of Outcomes of Severe Mental Disorders DOS) (Jablensky et al. 1992). Ziel dieser Arbeit war, Inzidenz und Prognose schwerer psychischer Erkrankungen, insbesondere der Schizophrenie, in verschiedenen Gesellschaften zu evaluieren. 12 Studienzentren nahmen an der Untersuchung teil: Aarhus (Dänemark), Agra und Chandigarh (Indien), 
Cali (Kolumbien), Dublin (Irland), Honolulu und Rochester (USA), Ibadan (Nigeria), Moskau (Russland), Nagasaki (Japan), Nottingham (Großbritannien) und Prag (Tschechien). Eingeschlossen wurden alle Erstkontakte mit Verdacht auf eine Erkrankung aus dem schizophrenen Formenkreis. Die Ergebnisse zeigten, dass bei Anwendung einer breiten Definition von Schizophrenie die jährlichen Inzidenzraten der Risikoaltersgruppe 15-54 Jahre zwischen 1,5 bis 4,2 pro 10.0oo Einwohner schwankten (Jablensky et al. 1992). Bei Verwendung enger gefasster Forschungskriterien der Schizophrenie lag die Spannbreite der jährlichen Inzidenzraten unter den beteiligten Zentren nur noch zwischen 0,7 bis 1,4 pro 10.00o Einwohner. Die Ergebnisse der DOS-Studie bestätigten die Resultate anderer epidemiologischer Untersuchungen, die in der Vergangenheit in verschiedenen Ländern durchgeführt wurden (Tseng 2001).

Von kulturanthropologisch orientierten Psychiatern wurde allerdings häufig moniert, dass die Autoren dieser Studie Gemeinsamkeiten hervorhoben und Unterschiede ignorierten. Zudem seien die epidemiologischen Daten der WHO-Studien aus den außereuropäischen Ländern vorwiegend in Großstädten wie Ibadan erhoben worden, weshalb über das Vorkommen von Schizophrenie in traditionellen segmentierten Gesellschaften keine Aussage getroffen werden könne. Eine Bestätigung fand diese Ansicht in aktuellen Arbeiten, die ein differenzierteres Licht auf die „epidemiologische Landschaft“ der Erkrankung werfen. In einer Metaanalyse von 161 Studien aus 33 Ländern fanden McGrath et al. (2004) doch eine deutliche Schwankungsbreite der Inzidenz (o,7 bis 4,3 pro 10.0oo Einwohner).

\section{Prävalenz}

Pfeiffer (1994) belegte durch zahlreiche Beispiele, dass akute und chronische schizophrene Psychosen auch in isolierten traditionellen Gesellschaften vorkommen. Im Vergleich zu den Inzidenzraten sind die Unterschiede der Prävalenz manifester schizophrener Krankheitsbilder in verschiedenen Populationen deutlicher (Sartorius et al. 1996). Eine neuere Metaanalyse von $188 \mathrm{Stu}-$ dien aus 46 Ländern bestätigte die Ergebnisse der WHO-Studien (Saha et al. 2005). Unabhängig vom Typ der Prävalenz (Punkt-, Perioden-, Lifetime-Prävalenz) zeigen sich deutliche interkulturelle Schwankungen. Die geschätzte Prävalenz der am „wenigsten entwickelten“ Länder (Median = 2,62 auf 1.00o Einwohner) ist statistisch signifikant niedriger $(\mathrm{p}=.02)$ als diejenige der „Schwellenländer“ (Median = 4,69 auf 1.00o Einwohner) und ,entwickelten“ Länder (Median = 3,30 auf 1.0oo Einwohner). Dass sich traditionelle Gemeinschaften von Übergangsgesellschaften unterscheiden, bestätigt die Ergebnisse der Feldforschungen von Torrey et al. (1974) in Papua Neu Guinea. Bei den abgeschieden lebenden Stämmen der Bergregionen fand sich eine Prävalenz zwischen o,03 und o,19 pro 1.00o Einwohner, in den Küstenbezirken, deren Bevölkerung seit langem regelmäßigen Kontakt zu Australiern und Europäern hatte, war die Prävalenzrate deutlich höher (o,38-0,77 pro 1.0oo). Darüber hi- 
naus konnte nachgewiesen werden, dass Inzidenz und Prävalenz der Schizophrenie mit dem Breitengrad variieren (Saha et al. 2006). Für beide Geschlechter stieg die Prävalenz mit dem Breitengrad an, bei der Inzidenz zeigte sich dieses Phänomen nur bei Männern. Die Interpretationsmöglichkeiten dieser Ergebnisse sind komplex und vielfältig. So könnte das häufigere Vorkommen von blanden und daher schwierig zu diagnostizierenden Residualzuständen in traditionsbestimmten agrarischen Milieus eine Rolle spielen (Pfeiffer 1994). Cooper et al. (1972) verweisen auf den möglichen Einfluss unterschiedlicher Taxonomien auf die Diagnosestellung und damit auf die daraus resultierende Prävalenz. Nicht zuletzt sollte die Drift-Hypothese (Dunham 1965) berücksichtigt werden, wonach schizophrene Patienten in schlechteren Wohngebieten leben, während vitalere Teile der Bevölkerung abwandern.

Ein bekanntes Beispiel für den Einfluss der Heredität sind die Ergebnisse der epidemiologischen Untersuchungen von Dale (1981) in Mikronesien. Zwischen den Jahrhunderte lang voneinander isolierten Inselgesellschaften differierte trotz der gemeinsamen evolutionsbiologischen Abstammung die Prävalenzrate für Schizophrenie deutlich (bezogen auf 1.ooo Einwohner ab dem 16. Lebensjahr) zwischen 9,7 (Yap) und o,8 (Marshall-Inseln). Für die höheren Prävalenzen auf Yap wurden in erster Linie die dort vorherrschenden endogamen Heiratssysteme als Erklärung herangezogen. Wie die Untersuchungen von Murphy und Raman (1971) auf Mauritius zeigten, können soziokulturelle Faktoren Einfluss auf die Chronifizierung der Erkrankung haben und damit ebenfalls zu unterschiedlichen Prävalenzraten führen (siehe unten). Diese Resultate stimmen mit Ergebnissen der Follow-up Studie der International Pilot Study of Schizophrenia (IPSS) der WHO (1979) überein, wonach schizophrene Patienten in Entwicklungsländern trotz nachteiliger Behandlungsbedingungen bessere Prognosen haben als Kranke in entwickelten Ländern (Sartorius et al. 1977, 1978). Pfeiffer (1994) führte zudem eine Reihe von Beispielen an, die sich nicht schlüssig durch Selektion erklären lassen: ungewöhnlich niedrige Prävalenzraten fanden sich beispielsweise bei den Hutteriten, bei indigenen Gruppen auf Taiwan und auf Tonga im Pazifik, während hingegen Iren eine besonders hohe Prävalenz schizophrener Störungen aufweisen (Murphy 1982).

Die Metaanalyse (Saha et al. 2005) bestätigte diese Ergebnisse. Es zeigte sich, dass Schizophrenie in Entwicklungsländern seltener vorkommt als in modernen Staaten und Schwellenländern. Die meisten neueren epidemiologischen Untersuchungen deuten also darauf hin, dass regionale Unterschiede existieren. Offen ist allerdings, ob es sich dabei um biologische Faktoren oder soziokulturelle Umstände handelt, die Vulnerabilitäten verstärken oder protektiv wirksam sind.

\subsubsection{Verlauf und Ausgang}

Die 2-Jahres Follow-up Untersuchung der International Pilot Study of Schiophrenia (WHO 1973) ergab günstigere klinische Verläufe und bessere Prognosen in den 
Entwicklungsländern (Sartorius et al. 1977). Eine weitere drei Jahre darauf folgende Katamnese bestätigte dieses Resultat (Leff et al. 1992; Sartorius et al. 1996; Tseng 2001). Kritiker gaben allerdings zu bedenken, dass hier unter Umständen ein diagnostisches Artefakt vorliegen könnte. Spätestens seit den 1950er-Jahren ist nämlich bekannt, dass kurz dauernde, akut verlaufende Psychosen in Afrika und Asien deutlich häufiger vorkommen als in Europa und Nordamerika (siehe oben). Dieser Einwand konnte allerdings durch die Autoren der erwähnte Determants of Outcome of Severe Mental Disorders Studie (DOS) der WHO weitgehend entkräftet werden (Jablensky et al. 1992). Diese konnten zeigen, dass Krankheitsverläufe und soziale Anpassung auch unter Verwendung eines sehr engen Schizophreniebegriffs in den außereuropäischen Ländern deutlich günstiger ausfielen als in Europa und der Vereinigten Staaten. Die Ergebnisse der DOS konnten in mehreren außereuropäischen Studien repliziert werden (Leon 1989; Verghese et al. 1989; Ohaeri 1993; Thara et al. 1994).

Ähnlich wie in den WHO-Studien fanden sich auch in der International Study on Psychotic Symptoms (ISPS) episodische Verläufe mit kompletten Remissionen häufiger in den beiden westafrikanischen Staaten (Nigeria: 43,5\%, Ghana: $51,3 \%$ ), chronische Verläufe dagegen häufiger in den europäischen (Österreich: $38 \%$, Polen: $23,7 \%$, Litauen: $20,6 \%$ ), besonders aber auch in den beiden asiatischen Staaten (Georgien: 39,2\%, Pakistan: 50,5\%) (s. Abb. 6).

In den letzten Jahren fanden sich abermals Hinweise auf methodische Mängel der Outcome-Studien (Patel 2006; Cohen et al. 2008). Die Varianz der Ergeb-

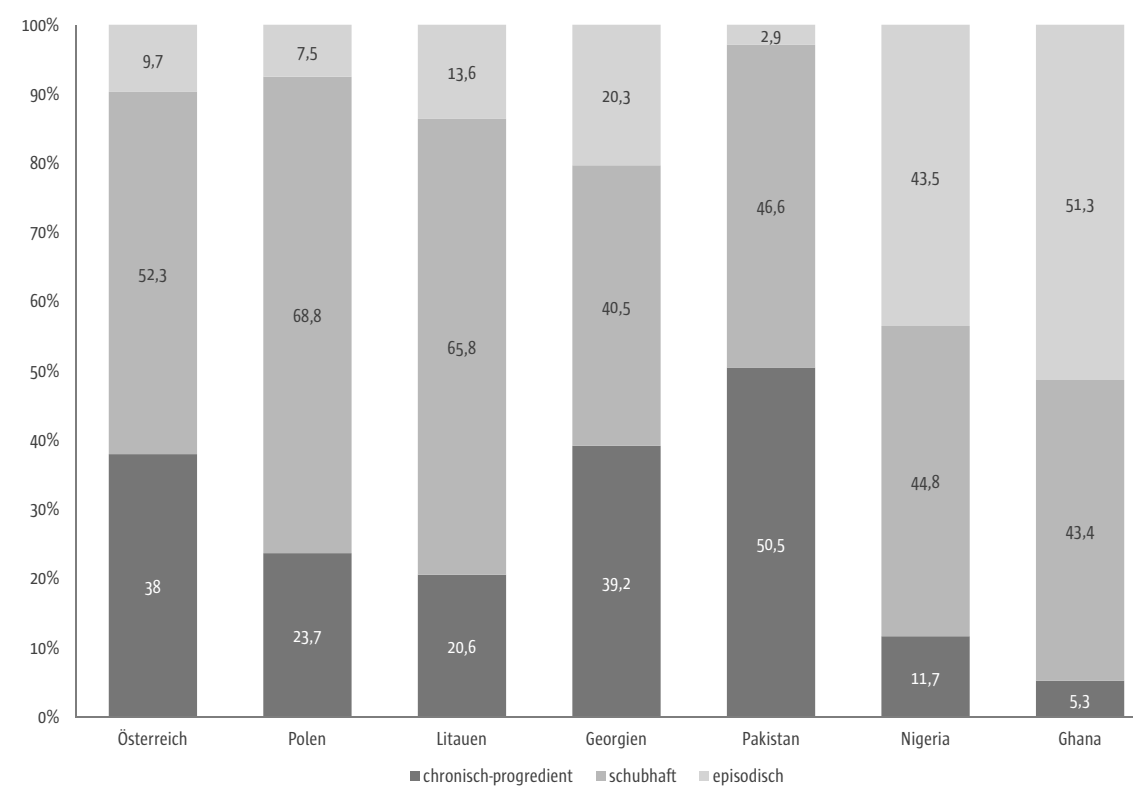

Abb. 6 Verlaufstypus der Schizophrenie (\%) in Österreich $(n=350)$, Polen $(n=80)$, Litauen $(n=73)$, Georgien $(n=74)$, Pakistan $(n=103)$, Nigeria $(n=324)$ und Ghana $(n=76)$ 
nisse zwischen den einzelnen Untersuchungen in nicht-westlichen Staaten sei groß. Darüber hinaus seien lokale Unterschiede in der Mortalität und Suizidalität von Schizophrenen bisher nicht ausreichend berücksichtigt worden. Da in vielen außereuropäischen Ländern psychiatrische Institution in nur unzureichenden Maße zur Verfügung stehen, gehören gerade Schizophreniekranke mit chronischen Verläufen zu den Teilen der Bevölkerung, die eine erhöhte Mortalitätsrate aufgrund des Selbstfürsorgedefizits aufweisen.

Die Befürworter der These von den besseren Krankheitsverläufen der Schizophrenie in traditionellen Kulturen wiederum vermuteten, dass - neben biologischen und individuellen Gründen - familiäre und soziokulturelle Faktoren dafür verantwortlich seien (Sartorius et al. 1978):

- Schutz und soziale Absicherung durch eine Großfamilie

- größere Akzeptanz der Umwelt gegenüber psychotisch Kranken und folglich Aufrechterhaltung der sozialen und emotionalen Kontakte

- besseres Integrationsvermögen traditioneller agrarischer Gesellschaften

Diese Erkenntnisse stehen in Einklang mit Ergebnissen aus der transkulturellen Forschung über expressed emotion ( $\mathrm{EE}=$ Kritik, Feindseligkeit und emotionales Überengagement in der unmittelbaren Umgebung des schizophren erkrankten Individuums), die nahe legen, dass Verlauf und Prognose schizophrener Erkrankungen mit dem soziokulturellen Umgang mit Emotionen in Verbindung stehen. In hoch industrialisierten Ländern wie England oder USA scheint die Häufigkeit von Familien mit hohen EE-Werten signifikant größer zu sein (England: 48\%, USA: 67\%) als in weniger industrialisierten Gesellschaften (Indien: 23\%) (Kavanagh 1992; Weisman et al. 2000). Dass die günstigeren Verläufe allerdings nur bedingt durch das familiäre Umfeld und die Sozialisationsbedingungen erklärt werden können, zeigen unter anderem die Ergebnisse der ISPS (s. Abb. 6). Bei vergleichbaren soziokulturellen Rahmenbedingungen (traditionelle Familiensysteme, agrarische Wirtschaft, tribale oder hierarchisch stratifizierte Gesellschaften etc.) waren die Verläufe in den afrikanischen Staaten deutlich besser, in den asiatischen Staaten deutlich schlechter als in den drei europäischen Ländern. Hier könnten Unterschiede in der Versorgungslage und im Inanspruchnahmeverhalten eine ausschlaggebende Rolle spielen.

\subsubsection{Subtypen der Schizophrenie}

Die klassischen Subtypen der Schizophrenie, i.e. Schizophrenia simplex, Hebephrenie, Katatonie und paranoide Schizophrenie, wurden in bisher allen Kulturen beschrieben. Schon Emil Kraepelin (1904a, b) bemerkte, dass sich die klinischen Bilder der Dementia praecox in Indonesien und in Deutschland bezüglich ihrer grundlegenden Gestalt nur unwesentlich unterschieden. Differenzen fanden sich allerdings in der Häufigkeitsverteilung der Subtypen; so traten beispielsweise katatoniforme Bilder seltener in Indonesien als in Deutschland auf (Kraepelin 1904b; Bendick 1989; Boroffka 1989), ein Ergebnis, 
das mehrere Jahrzehnte später von Pfeiffer repliziert werden konnte (Pfeiffer 1994). Murphy et al. (1963) zeigten, dass in Asien und in Afrika der katatone Subtypus und die Schizophrenia simplex relativ häufig vorkommen, während die paranoide Verlaufsformen im Vergleich zu Europa und Nordamerika seltener waren. In der International Pilot Study of Schizophrenia war der paranoide Subtypus sowohl im gesamten Sample $(38,9 \%)$ als auch in den einzelnen Studienzentren - mit Ausnahme von Agra (Indien), Cali (Kolumbien) und Moskau (Russland) - am häufigsten verbreitet. Weltweit mit 13,2\% am zweithäufigsten war der schizoaffektive Subtypus, am seltensten die katatone Form mit insgesamt $6,7 \%$ vertreten. Relativ hohe Prävalenzen für die katatone Schizophrenie fanden sich in Agra (Indien) mit 22\%, in Cali (Kolumbien) mit 13\% und in Ibadan (Nigeria) mit 8,3\%. Historische Längsschnittuntersuchungen belegen, dass die Prävalenz der katatone Schizophrenie in den Industriegesellschaften seit dem Beginn des 20. Jahrhunderts kontinuierlich abgenommen hat, während parallel dazu der paranoide Subtypus häufiger registriert wurde (Stompe et al. 2002). In der International Study on Psychotic Symptoms (Stompe u. Friedmann 2007), die unter anderem die Verteilung der schizophrenen Subtypen und der schizophrenieähnlichen Psychosen in sieben Ländern (Georgien, Ghana, Litauen, Nigeria, Österreich, Pakistan, Polen) untersuchte, fanden sich die akuten, affektgetragenen Subtypen (schizoaffektiv, schizophreniform, kurze psychotische Störung) sowie die katatone Schizophrenie häufiger in den beiden afrikanischen Staaten, der desorganisierte Typus am häufigsten in Pakistan, in den europäischen Ländern dominierte hingegen deutlich der paranoide Typus.

\subsubsection{Symptomatik}

Während ursprünglich davon ausgegangen wurde, dass die Prävalenz der Schizophrenie primär von genetischen Faktoren abhängig ist, zeigte sich beim Vergleich der psychotischen Symptomatik, dass hier soziokulturellen Verhältnissen sowie interpersonell, in modernen Gesellschaften auch medial vermittelten Werte- und Symbolsystemen eine bedeutsame Rolle in der Ausgestaltung von Form und Inhalt zukommt (Bell et al. 2005; Gold u. Gold 2012; Lerner et al. 2006; Schmidt-Siegel et al. 2004; Stompe et al. 1999). Besonders bei den Inhalten von Wahn und Halluzinationen findet man eine hohe regionale Variabilität mit einer raschen Reaktion auf zeitgeschichtliche Ereignisse und technische Innovationen (Bauer et al. 2011; Kala u. Wig 1982; Kim et al. 2001; Murphy 1980; Murphy et al. 1963; Ndetei u. Singh 1982, 1983; Ndetei u. Vadher 1984a, b; Risso u. Böker 1967; Stompe u. Friedmann 2007; Stompe et al. 1999, 2006; Suhail u. Cochrane 2002; Tateyama et al. 1998). Dabei sollte allerdings nicht übersehen werden, dass es in allen untersuchten Gesellschaften einen stabilen Kern schizophrener Wahnthemen gibt, der mehr von den Grundbedingungen des psychotischen Erlebniswandels als von soziokulturellen Umfeldbedingungen abhängig ist (Stompe et al. 2007). Wenn man die Ergebnisse der vorliegenden Untersuchungen zusammenfasst, so ergibt sich folgendes 
Bild: visuelle Halluzinationen und religiöse Wahnideen finden sich häufiger in traditionellen Gesellschaften, Schuldwahn dagegen in christlich dominierten westlichen Ländern. Während in allen Ländern Verfolgungs- und Crößenwahn die häufigsten Inhalte sind, ist die konkrete Ausgestaltung (wer verfolgt mich? auf welche Weise? aus welchem Grund und mit welchem Ziel?) ganz überwiegend vom soziokulturellen Umfeld abhängig.

Wenn man diese Details bei Seite lässt und die schizophrenen Einzelsymptome zu übergeordneten, das klinische Bild dominierenden Verbänden zusammenschließt, zeigen sich noch immer deutliche Unterschiede, die jedoch nicht eindeutig interpretierbar sind (s. Abb. 7).

Die Daten der ISPS zeigen, dass das klinische Bild der europäischen Patienten mit Schizophrenie weitgehend durch das paranoid-halluzinatorische Syndrom geprägt ist (Österreich: $78,5 \%$, Polen: $87,6 \%$, Litauen: $90,4 \%$ ), während katatone Symptome und Denkstörungen vergleichsweise selten zu finden sind. Nun prägen auch in den asiatischen und den afrikanischen Ländern Wahn und Halluzinationen das klinische Bild, es zeigen sich allerdings bedeutungsvolle Unterschiede. In allen vier außereuropäischen Staaten waren schizophre-

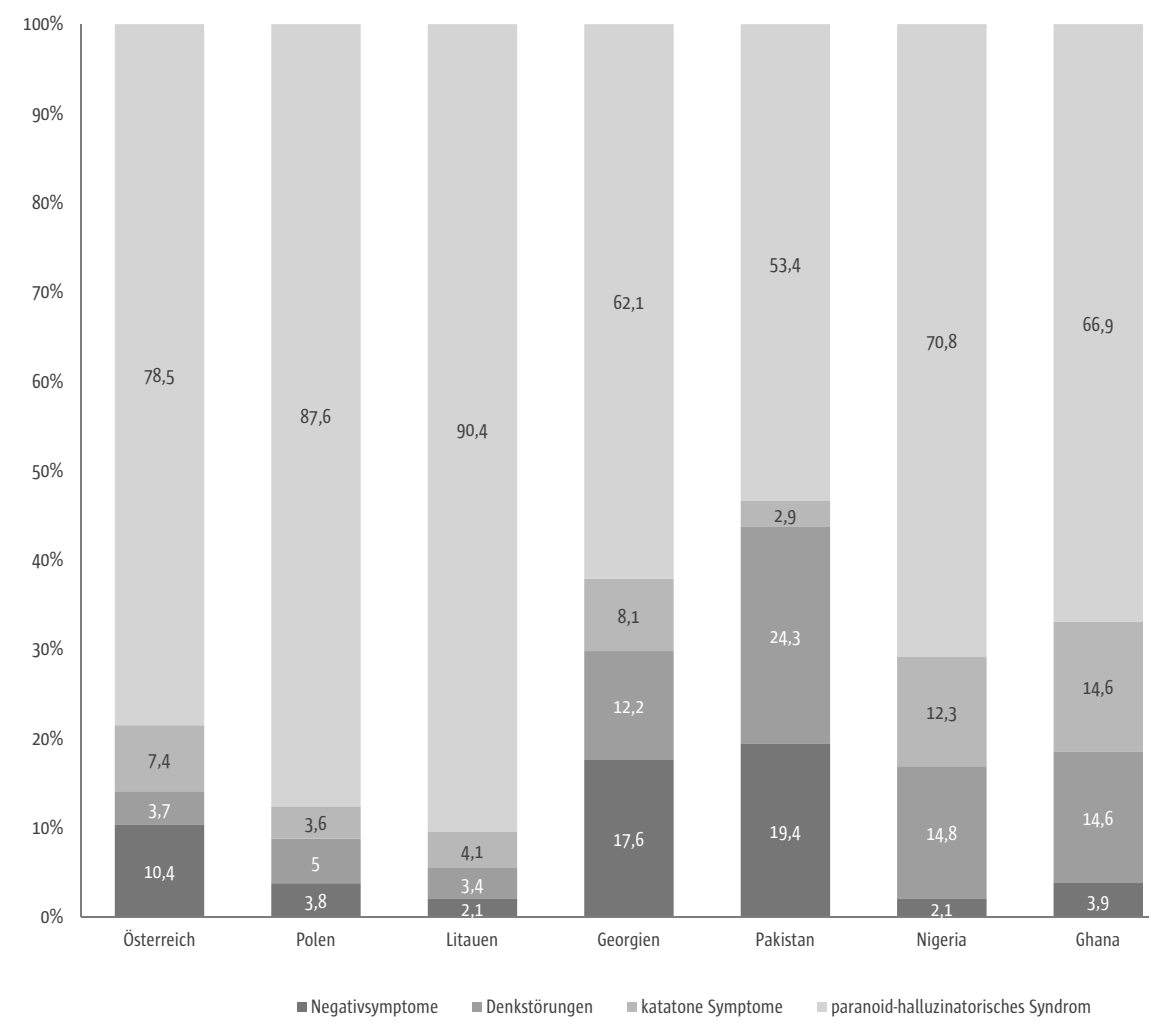

Abb. 7 Dominante schizophrene Symptomatik (\%) in Österreich $(n=350)$, Polen $(n=80)$, Litauen $(n=73)$, Georgien $(n=74)$, Pakistan $(n=103)$, Nigeria $(n=324)$ und Ghana $(n=76)$ 
nietypische Denkstörungen häufiger dominant. In Georgien und Pakistan fanden sich öfters Negativsymptome, in Nigeria und Ghana wurden hingegen häufiger das Krankheitsbild dominierende katatone Symptome beschrieben.

\subsubsection{Negative Diskriminierung schizophrener Menschen}

Trotz der Antistigma- und Empowermentbewegungen in den letzten 20 Jahren erfahren Schizophreniekranke noch immer zahlreiche Benachteiligungen in Beruf und Privatleben. Erlebte Stigmatisierung kann zu sozialem Rückzug führen, Behandlungs-und Betreuungseinrichtungen werden nicht oder nicht ausreichend in Anspruch genommen. Da die konsequente Behandlung aber Voraussetzung für einen günstigeren Krankheitsverlauf ist, kommt es bei Kranken, die sich als diskriminiert erleben, zu häufigeren Schüben und zur Chronifizierung der Erkrankung. Dies wiederum ist der Beginn eines Teufelskreises, an dessen Ende Verarmung, Beziehungslosigkeit und erhöhte Mortalität stehen.

Eine Arbeitsgruppe (INDIGO-Research-Network) untersuchte die erfahrene und erlebte Diskriminierung von schizophrenen Menschen in 27 Ländern (Thornicroft et al. 2009; Rose et al. 2011; Uçok et al. 2012). Es zeigte sich, dass die Rate der Stigmatisierung weltweit noch immer sehr hoch ist (s. Abb. 8).

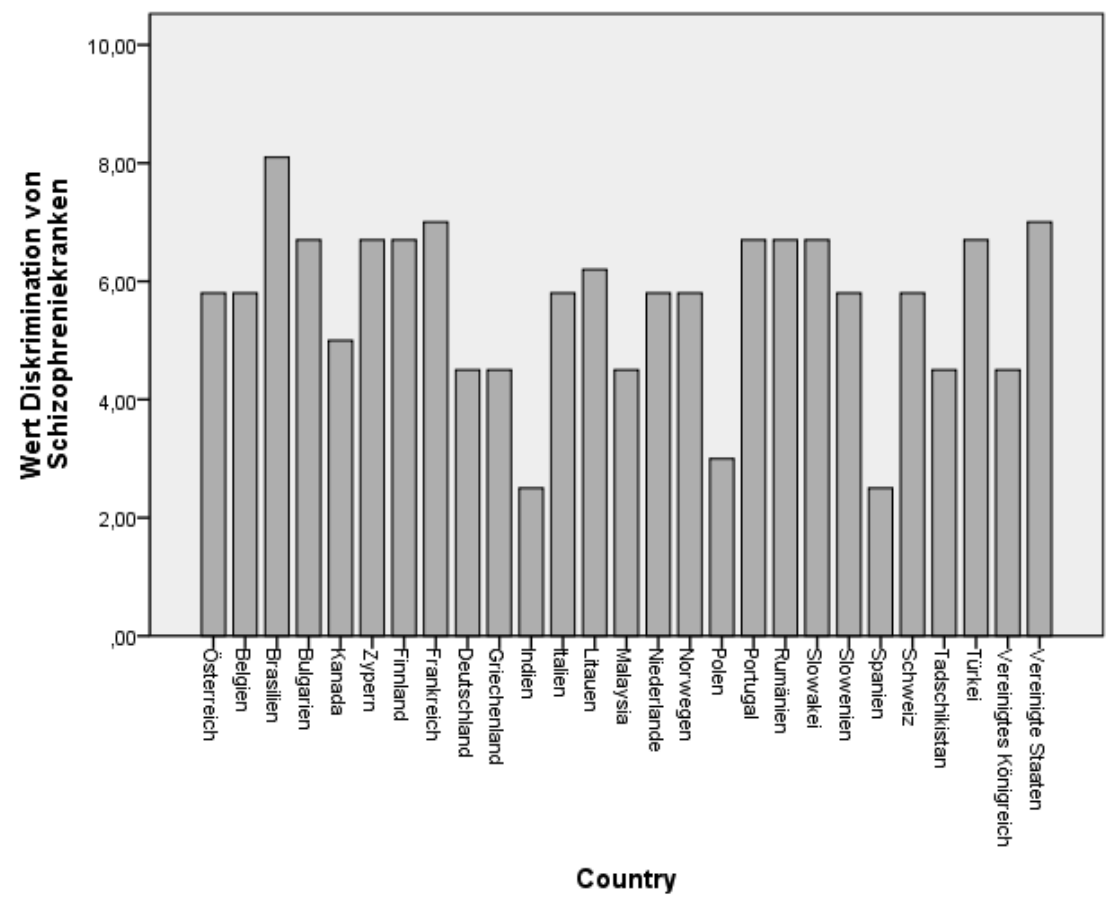

Abb. 8 Negative Diskriminierung schizophrener Menschen (nach Thornicroft et al. 2009) 
$47 \%$ von 729 Teilnehmern hatten aufgrund ihrer Erkrankung Schwierigkeiten, Freunde zu finden, 43\% fühlten sich von Familienmitgliedern zurückgesetzt, $29 \%$ hatten Probleme einen Job zu finden oder zu behalten, $27 \%$ berichteten über Diskriminierung durch (potenzielle) Intimpartner. Stärker noch als die reale Stigmatisierung wirkte sich die antizipierte Diskriminierung aus. $72 \%$ der Befragten fühlten sich angehalten, ihre Diagnose zu verschweigen, 64\% gehen schon mit negativen Vorannahmen auf Arbeitssuche, auch im Bereich Intimität und Sexualität nahmen 55\% eine Zurückweisung bereits vorweg als sicher an (Thornicroft et al. 2009). Neben diesen grundsätzlichen Trends finden sich allerdings auch deutliche lokale Unterschiede (s. Abb. 8). In Spanien, Polen und Indien erleben sich Schizophreniekranke als verhältnismäßig wenig stigmatisiert. Hohe Werte für negative Diskriminierung finden sich dagegen, um nur einige herauszugreifen, in so unterschiedlichen Ländern wie Brasilien, Bulgarien, Finnland, Portugal oder Rumänien. Offensichtlich besteht kein Zusammenhang zwischen der Stigmatisierung und dem Modernisierungsgrad oder der gesellschaftlichen Ungleichheit innerhalb der einzelnen Länder. Insgesamt zeigt sich aber, dass die Situation von Schizophreniekranken in Europa und Amerika etwas schlechter ist als in asiatischen Staaten (s. Abb. 9).

Dass damit allerdings nicht unbedingt bessere Verläufe verbunden sind, zeigen die Ergebnisse der ISPS, wo die beiden asiatischen Staaten den höchsten

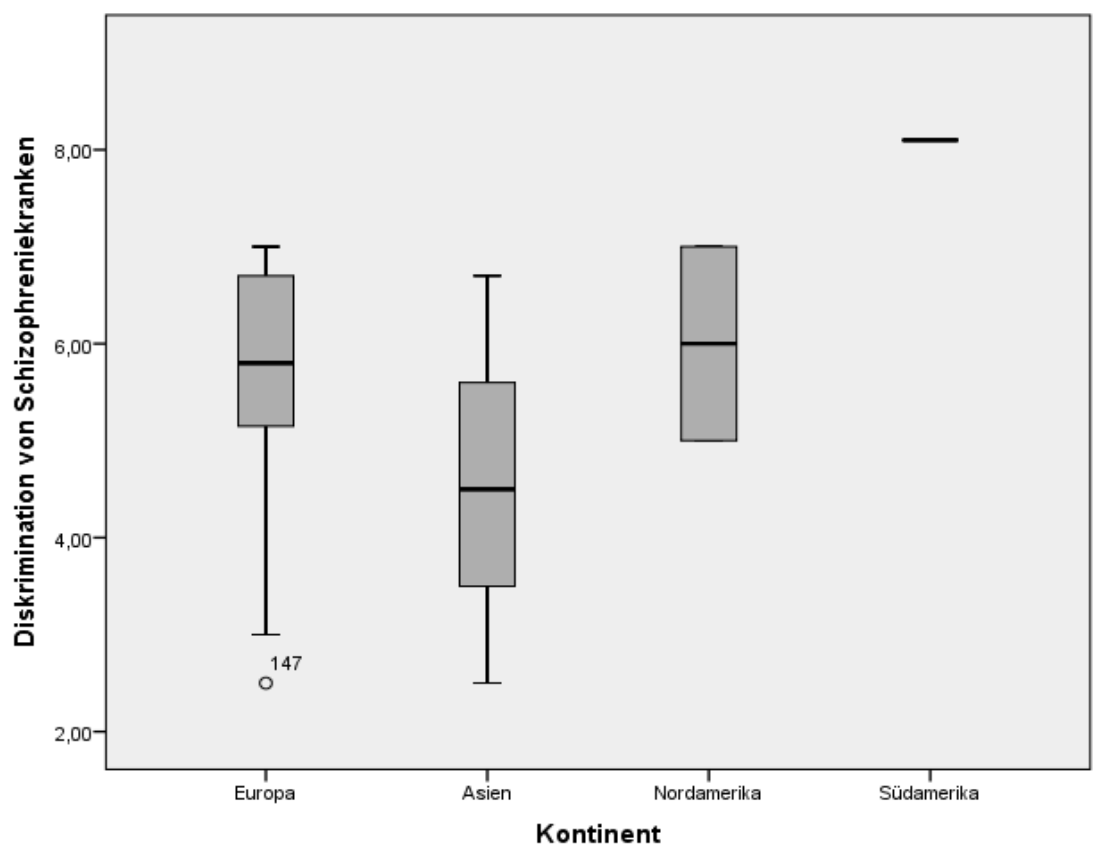

Abb. 9 Diskriminierung von schizophrenen Menschen nach Kontinenten (nach Thornicroft et al. 2009) 
Anteil an chronischen Verläufen (s. Abb. 6) mit ausgeprägter Negativsymptomatik (s. Abb. 7) aufweisen.

\subsection{Akute vorübergehende (transiente) Psychosen}

\subsubsection{Geschichte}

Transiente Psychosen sind eine heterogene, von den Schizophrenien und den manisch-depressiven Erkrankungen unterscheidbare Gruppe von Psychosen. Erstmals wurden sie unter dem Begriff bouffées délirantes (Magnan u. Legrain 1895) in Frankreich beschrieben. Auch in der deutschsprachigen Psychiatrie fanden sich in den folgenden Jahrzehnten ähnliche Darstellungen von akuten, in keine der gebräuchlichen Krankheitskategorien passenden Psychosen. Parallelen zu den Bouffées délirantes gibt es in den Beschreibungen der psychogenen Psychose von Jaspers (1973), der Emotionspsychosen von Labhard (1963), der mischbildhaften phasischen Psychosen von Mentzos (1967) und vor allem der zykloiden Psychosen von Leonhard (2003). Im skandinavischen Sprachraum wurden diese Krankheitsbilder unter dem Begriff der schizophreniformen Psychosen bekannt (Langfeldt 1939). Im ICD-1o der WHO (1993) entspricht der transiente Psychose die akute vorübergehende psychotische Störung (ICD10: F23), im DSM-IV der American Psychiatric Association (1996) die kurze psychotische Störung (DSM-IV: 298.9). Bereits in den fünfziger Jahren des vorigen Jahrhunderts wurde auf die Häufigkeit dieser Psychosen in der dritten Welt hingewiesen, eine Ansicht, die in den folgenden Jahrzehnten mehrfach bestätigt werden konnte (Jilek u. Jilek-Aall 1970).

\subsubsection{Symptomatik und Verlauf}

Transiente Psychosen dauern zumeist nur Stunden bis einige Wochen und zeichnen sich durch eine komplette Remission aus. Häufig finden sich auslösende Faktoren wie akuter psychischer Stress, Schwangerschaften und Geburt oder Drogenmissbrauch. Die Stimmungslage ist zumeist ängstlich, mitunter auch ekstatisch. Wenn die Angst im Vordergrund steht, berichten die Patienten retrospektiv über Misstrauen, Eigenbeziehungen, hypochondrische Ideen, Minderwertigkeitsideen, Selbstvorwürfe und Entfremdungserlebnisse, optische Illusionen und Halluzinationen, Geruchshalluzinationen, körperliche Missempfindungen und Beeinflussungserlebnisse. Im Extremfall kann ein traumhaft-oneiroidaler Erlebniswandel auftreten. Der körperliche Ausdruck der Angst reicht von höchster Erregung bis zur Bewegungslosigkeit. Dominiert die Ekstase, kommt es häufig zu Beglückungs- bzw. Erlösungsideen. Nicht selten stehen allerdings weniger affektive Veränderungen, sondern mehr akute Störungen des Denkens oder der Psychomotorik im Vordergrund. Dominieren Denkstörungen, kommt es häufig zu einem Wechsel zwischen ratlosem Stupor und verworrener Erregung mit oft bizarren Wahneinfällen, Personen- 
verkennungen, Beziehungsideen, akustischen Halluzinationen oder Bedeutungsideen. Die Erregung führt zu einer Inkohärenz des Denkens mit Rededrang. Steht die Denkhemmung im Vordergrund, zeigt sich eine Sprachverarmung, die bis zum Mutismus führen kann. Dominieren die Störungen der Psychomotorik, findet sich im Zustand starker Erregung eine quantitative Steigerung der Ausdrucks- oder Reaktivbewegungen. Die Patienten gestikulieren, winken oder bieten ein vielfältiges Mienenspiel. Unartikuliertes Schreien oder Johlen ist häufig. In akinetischen Phasen kommt es regelhaft zur Aufhebung der Reaktivbewegungen und zu einer Erstarrung der Ausdrucksmotorik, die Haltung ist steif, das Gesicht starr. Gelegentlich sind Haltungsverharren oder Gegenhalten nachweisbar.

\subsubsection{Vorkommen}

Transiente Psychosen sind in zahlreichen Gesellschaften beschrieben worden. Sie scheinen allerdings in traditionellen Ethnien und Kulturen häufiger als in modernen Industriegesellschaften vorzukommen. Schilderungen finden sich zum Beispiel bei Patienten aus Afrika (Carothers 1953; Collomb 1965; Field 1958), Indonesien (Pfeiffer 1994), den Antillen (Despinoy u. Camelio 1967) oder bei den Mohave-Indianern (Devereux 1961).

\section{Ausblick: Migrationspsychiatrische Problemstellungen}

In der Diagnosestellung einer Psychose bei Patienten aus anderen Kulturen findet sich ein weites Spektrum möglicher Fehldiagnosen. Mangelnde kulturelle Kompetenz und Sprachbarrieren aufseiten der Untersucher ergeben oft eine Überschätzung der Häufigkeit schizophrener Erkrankungen bei Migranten in Krankenhauspopulationen (z.B. Haasen et al. 1997; Holzmann et al. 1994; Machleidt u. Calliess 2005).

Nach Cochrane und Bal (1987) führen zwei Faktoren häufig zu Fehldiagnosen:

1. In nicht-europäischen Kulturen finden sich psychotische Symptome häufig auch in Form akuter Stress- und Belastungsreaktionen sowie bei Angststörungen oder depressiven Erkrankungen (Pfeiffer 1994). Insbesondere Halluzinationen und paranoide Symptome treten häufig auch bei bipolaren affektiven Erkrankungen oder monopolaren Manien auf. Ebenso sind psychotische Reaktionsbildungen bei neurotischen Störungen sind nicht selten anzutreffen (Ndetei u. Singh 1982, 1983).

2. Die häufig schwierige Differenzierung zwischen Wahn und Glaube sowie zwischen Halluzinationen und Trance kann im interkulturellen Kontext zu erheblichen Fehlbeurteilungen führen (s.a. Pfeiffer 1994; Murphy 1980; Westermeyer 1987). 
Haasen et al. (2005) schlugen daher folgende Leitlinien zur psychopathologischen Beurteilung psychotischer Störungen im Kulturvergleich vor:

- Bei Patienten aus fremden Kulturkreisen dürfen psychotische Symptome nicht vorschnell mit einer schizophrenen Störung assoziiert werden. Die Möglichkeit einer stressbedingten Genese muss differenzialdiagnostisch stärker in Betracht gezogen werden als Patienten euro-amerikanischer Herkunft.

- Unabhängig von kulturellen Einflussfaktoren gibt es ubiquitäre Kernsymptome der Schizophrenie. Allerdings ist es unzulässig, die Diagnose einer schizophrenen Störung bei Patienten aus fremden Kulturkreisen aufgrund des Vorhandenseins nur eines (!) Symptoms zu stellen, insbesondere dann, wenn es sich um Wahnphänomene oder Halluzinationen handelt. Das psychosoziale Funktionsniveau und Negativsymptomatik müssen bei der Beurteilung Berücksichtigung finden.

- Die psychopathologische Beurteilung psychotischer Symptomatik kann durch kulturelle Faktoren erheblich erschwert sein. Die Differenzierung zwischen Wahn und Glaube sowie zwischen Halluzination und Trance muss im kulturellen Kontext, unter Umständen unter Zuhilfenahme fremdanamnestischer Daten, erfolgen.

- Gleichzeitig dürfen aber kulturell gefärbte Wahnideen nicht vorschnell als kulturspezifischer Glaube eingeordnet werden. Bestimmte Gedanken können zwar im Kulturkreis des Patienten glaubensbedingt sein, trotzdem aber eine pathognomonische Wertigkeit bekommen. In Zweifelsfällen ist ein Vergleich mit der Vorstellungswelt der Angehörigen hilfreich.

- Paranoide Wahnideen müssen gründlich auf ihren realen Kontext hin überprüft werden. Insbesondere Flüchtlinge geraten häufig aufgrund ihrer leidvollen, unter Umständen traumatischen Erfahrungen in paranoide Übertragungskonstellationen.

- Muttersprachliche Behandler oder professionelle Dolmetscher sollen als Kulturmediatoren zu Hilfe genommen werden.

\section{Literatur}

American Psychiatric Association (1996) Diagnostisches und statistisches Manual psychischer Störungen. DSM-IV. Hogrefe Göttingen Bern Toronto Seattle

Bauer S, Schanda H, Karakula H, Rudaleviciene P, Okribelashvili N, Chaudhry HR, Idemudia I, Gscheider S, Ritter K, Stompe T (2011) Culture and the prevalence of hallucinations in schizophrenia. Compr Psychiatry 52, 319-325

Bell V, Grech E, Maiden C, Halligan PW, Ellis HD (2005), Internet delusions': a case series and theoretical integration. Psychopathology 38, 144-150

Bendick CH (1989) Emil Kraepelins Forschungsreise nach Java im Jahre 1904. In: Arbeiten der Forschungsstelle des Instituts für Geschichte der Medizin. Band 49. Kohlhauer Köln

Bleuler E (1911) Dementia praecox oder Gruppe der Schizophrenien. Deuticke Leipzig Wien

Boroffka A (1989) Emil Kraeplin (1856-1926). Transcultural Psychiatric Research Review 27, 228-237 
Carothers IC (1953) The African Mind in Health and Disease. A Study of Ethnopsychiatry. World Health Organization Press Geneva

Cochrane R, Bal S (1987) Migration and schizophrenia: an examination of five hypotheses. Soc Psychiatry 22, 181-191

Cohen A, Patel V, Thara R, Gureje 0 (2008) Questioning an axiom: better prognosis for schizophrenia in the developing world? Schizophr Bull 34, 229-244.

Collomb H (1965) Bouffées délirantes en psychiatrie Africaine. Psychopathologie africaine 1, 167-239

Cooper JE, Kendell RE, Gurland BI, Sharpe L, Copeland IRM, Simon R (1972) Psychiatric Diagnosis in New York and London. Oxford University Press London

Dale PW (1981) Prevalence of schizophrenia in the Pacific island populations of Micronesia. J Psychiatr Res 16, $103-111$

Despinoy M, Camelio A (1967) La psychopathologie aux Antilles et ses relations avec ses structures sociales. In: Petrilowitsch N (Hrsg.) Beiträge zur vergleichenden Psychiatrie. Band 1. 272-298. Karger Basel

Devereux G (1961) Mohave ethnopsychiatry and suicide. The psychiatric knowlege and the psychiatric disturbancies of an Indian tribe. 175. Smithsonian Inst Bureau Amer Ethnology Bull Washington

Devereux G (1985) Realität und Traum: Psychotherapie eines Prärie-Indianers. Suhrkamp Frankfurt am Main

Dunham HW (1965) Community and Schizophrenia: an Epidemiological Analysis. Wayne State University Press Detroit

Edgerton RB, Cohen A (1994) Culture and schizophrenia: the DOSMD challenge. Br J Psychiatry 164, 222-231

Field MJ (1958) Mental disorder in rural Ghana. J Ment Sci 104, 1034-1051

Gold I, Gold I (2012) The „Truman Show“ delusion: psychosis in the global village. Cogn Neuropsychiatry 17,455-472

Haasen C, Kleinemeier E, Yagdiran 0 (2005) Kulturelle Aspekte bei der Diagnostik psychischer Störungen. In: Assion HJ (Hrsg.) Migration und seelische Gesundheit. 145-156. Springer Heidelberg

Haasen C, Lambert M, Yagdiran 0, Krausz M (1997) Psychiatric disorders among migrants in Germany: prevalence in a psychiatric clinic and implications for services and research. Eur Psychiatry 12, 305-310

Holzmann TH, Volk S, Georgi K, Pflug B (1994) Ausländische Patienten in stationärer Behandlung in einer psychiatrischen Universitätsklinik mit Versorgungsauftrag. Psychiat Prax 21, 106-108

Hopper K, Harrison G, Janca A, Sartorius N (2001) Recovery from schizophrenia: An international perspective. Oxford University Press New York

Jablensky A, Sartorius N, Ehrenberg G, Anker M, Korten A, Cooper JE, Day R, Bertelsen A (1992) Schizophrenia: manifestations, incidence and course in different cultures: A World Health Organisation Ten Countries Study (Psychological Medicine, Monograph Supplement No. 20). Cambridge University Press Cambridge UK laspers K (1973) Allgemeine Psychopathologie. 9. Auflage. Springer Berlin Heidelberg New York jilek WG, Jilek-Aall L (1970) Transient psychoses in Africans. Psychiat Clin 3, 311-324

Kala AK, Wig NN (1982) Delusions across cultures. Int I Soc Psychiatry 28; 185-193

Kavanagh DJ (1992) Recent developments in expressed emotion and schizophrenia. Br | Psychiatry 160, 601-620 Kim K, Hwu H, Zhang LD, Lu MK, Hwang TJ, Kim D, Park YC (2001) Schizophrenic delusions in Seoul, Shanghai and Taipei: a transcultural study. J Kor Med Sci 16, 88-94

Kraepelin E (1904a) Vergleichende Psychiatrie. Zentralbl Nervenheilk Psychiatr 27, 433-437

Kraepelin E (1904b) Psychiatrisches aus Java. Zentralbl Nervenheilk Psychiatr 27, 468-469

Labhardt F (1963) Die schizophrenieähnlichen Emotionspsychosen. Ein Beitrag zur Abgrenzung schizophrenieähnlicher Zustandsbilder. Springer Berlin Göttingen Heidelberg

Lambo TA (1957) Some unusual features of schizophrenia among primitive peoples. West Afr Med I 6, 147-152 Langfeldt $G$ (1939) The schizophreniform states. Munksgaard Copenhagen

Leff J, Sartorius N, Jablensky A, Korten A, Ernberg G (1992) The International Pilot Study of Schizophrenia: fiveyear follow-up findings. Psychol Med 22, 131-145

León CA (1989) Clinical course and outcome of schizophrenia in Cali, Colombia. A 10-year follow-up study. I Nerv Ment Dis 177, 593-606

Leonhard K (2003) Aufteilung der endogenen Psychosen und ihre differenzierte Ätiologie. 8. Auflage. Thieme Stuttgart New York

Lerner V, Libov I, Witztum E (2006) „Internet delusions“: the impact of technological developments on the content of psychiatric symptoms. Isr | Psychiatry Relat Sci 43(1),47-51 
Machleidt W, Calliess IT (2005) Transkulturelle Psychiatrie und Migration - Psychische Erkrankungen aus ethnischer Sicht. Die Psychiatrie 2, 77-84

Magnan V, Legrain M (1895) Les dégénérés. État mentale et syndromes épisodiques. Rueff et Cir Paris

McGrath J, Saha S, Welham I, El Saadi O, MacCauley C, Chant D (2004) A systematic review of the incidence of schizophrenia: the distribution of rates and the influence of sex, urbanity, migrant status and methodology. BMC Med 28, 13

Mentzos S (1967) Mischzustände und mischbildhafte phasische Psychosen. Enke Stuttgart

Murphy HBM (1980) Kulturelle Aspekte des Wahns. In: Pfeiffer WM, Schoene W (Hrsg.) Psychopathologie im Kulturvergleich. 85-101. Ferdinand Enke Verlag Stuttgart

Murphy HBM (1982) Comparative Psychiatry. Springer Berlin Heidelberg New York

Murphy HBM, Raman AC (1971) The chronicity of schizophrenia in indigenous tropic people. Br I Psychiatry 118, $489-497$

Murphy HBM, Wittkower ED, Fried I, Ellenberger HF (1963) A cross-cultural survey of schizophrenic symptomatology. Int / Soc Psychiatry 9, 237-249

Ndetei DM, Singh A (1982) A study of delusions in Kenyan schizophrenic patients diagnosed using a set of research diagnostic criteria. Acta Psychiatr Scand 66, 208-215

Ndetei DM, Singh A (1983) Hallucinations in Kenyan schizophrenic patients. Acta Psychiatr Scand 67, 144-147

Ndetei DM, Vadher A (1984a) Frequency and clinical significance of delusions across cultures. Acta Psychiatr Scand 70, 73-76

Ndetei DM, Vadher A (1984b) A comparative cross-cultural study of the frequencies of hallucinations in schizophrenia. Acta Psychiatr Scand 70, 545-549

Ohaeri JU (1993) Long-term outcome of treated schizophrenia in a Nigerian cohort. Retrospective analysis of 7-year follow-ups. I Nerv Ment Dis 181, 514-516

Patel V, Cohen A, Thara R, Gureje 0 (2006) Is the outcome of schizophrenia really better in developing countries? Rev Bras Psichiatr 28, 149-152

Pfeiffer W (1994) Transkulturelle Psychiatrie. Thieme Stuttgart New York

Radley II, Morrison JH (2005) Repeated stress and structural plasticity in the brain. Ageing Res Rev 4, 271-287

Risso M, Böker W (1967) Der Einfluss des magischen Weltbildes auf die Gestaltung geistiger Störungen bei süditalienischen Patienten. In: Petrilowitsch N (Hrsg.) Beiträge zur vergleichenden Psychiatrie. Basel Karger

Rose D, Willis R, Brohan E, Sartorius N, Villares C, Wahlbeck K, Thornicroft G (2011) INDIGO Study Group. Reported stigma and discrimination by people with a diagnosis of schizophrenia. Epidemiol Psychiatr Sci. 20, 193-204

Saha S, Chant D, Welhalm J, McGrath I (2005) A systematic review of the prevalence of schizophrenia. PLoS Med 2, 413-433

Saha S, Chant D, Welhalm I, McGrath || (2006) The incidence and prevalence of schizophrenia varies with latitude. Acta Psychiatr Scand 114, 36-39

Sartorius N, Gulbinat W, Harrison G, Laska E, Siegel C (1996) Long-term follow-up of schizophrenia in 16 countries: A description of the International Study of Schizophrenia conducted by the World Health Organization. Soc Psychiatry Psychiatr Epidemiol 31, 249-258

Sartorius N, Jablensky A, Shapiro R (1977) Two-year follow-up of the patients included in the WHO International Pilot Study of Schizophrenia. Psychol Med 7, 529-541

Sartorius N, Jablensky A, Shapiro R (1978) Cross-cultural differences in the short term prognosis of schizophrenic psychoses. Schizophr Bull 4, 102-113

Sartorius N, Shapiro R, Kimura M, Barrett K (1972) WHO international pilot study of schizophrenia. Psychol Med 2, 422-425

Schmid-Siegel B, Stompe T, Ortwein-Swoboda G (2004) Being a webcam. Psychopathology 37, 84-85

Schneider K (1952) Klinische Psychopathologie. Thieme Stuttgart

Stompe T, Bauer S, Karakula H, Rudaleviciene P, Okribelashvili N, Chaudhry HR, Idemudia EE, Gscheider S (2007) Paranoid-hallucinatory Syndromes in Schizophrenia - Results of the International Study on Psychotic Symptoms. WCPRR 4, 63-68

Stompe T, Bauer S, Ortwein-Swoboda G, Schanda H, Karakula H, Rudalevicienne P, Chaudhry HR, Idemudia ES, Gschaider S (2006) Delusions of guilt: The attitude of Christian and Islamic confessions towards Good and Evil and the responsibility of men. I Muslim Ment Health 1, 43-56 
Stompe T, Friedmann A (2007) Culture and Schizophrenia. In: Bhugra D, Bhui K (Hrsg.) Textbook of Cultural Psychiatry. 314-323. Cambridge University Press Cambridge

Stompe T, Friedmann A, Ortwein G, Strobl R, Chaudhry HR, Najam N, Chaudhry MR (1999) Comparison of delusions among schizophrenics in Austria and in Pakistan. Psychopathology 32, 225-234

Stompe T, Ortwein-Swoboda G, Ritter K, Schanda H, Friedmann (2002) Are we witnessing the disappearance of catatonic schizophrenia? Compr Psychiatry 3, 167-174

Suhail LK, Cochrane R (2002) Effect of culture and environment on the phenomenology of delusions and hallucinations. Int | Soc Psychiatry 48, 126-138

Tateyama M, Asai M, Hashimoto M, Bartels M, Kaspers S (1998) Transcultural study of schizophrenic delusions. Tokyo versus Vienna and Tübingen (Germany). Psychopathology 31, 59-68

Thara R, Henrietta M, Joseph A, Rajkumar S, Eaton WW (1994) Ten-year course of schizophrenia-the Madras longitudinal study. Acta Psychiatr Scand 90, 329-336

Thornicroft G, Brohan E, Rose D, Sartorius N, Leese M (2009) INDIGO Study Group. Global pattern of experienced and anticipated discrimination against people with schizophrenia: a cross-sectional survey. Lancet 373(9661), 408-15

Torrey EF, Torrey B, Burton-Bradley BG (1974) The epidemiology of schizophrenia in Papua New Guinea. Am J Psychiatry 131, 567-573

Tseng WS (2001) Handbook of Cultural Psychiatry. Academic Press San Diego London Boston New York Sydney Tokyo Toronto

Uçok A, Brohan E, Rose D, Sartorius N, Leese M, Yoon CK, Plooy A, Ertekin BA, Milev R, Thornicroft G (2012) INDIGO Study Group. Anticipated discrimination among people with schizophrenia. Acta Psychiatr Scand 125(1), 77-83

Verghese A, John JK, Rajkumar S, Richard J, Sethi BB, Trivedi JK (1989) Factors associated with the course and outcome of schizophrenia in India. Results of a two-year multicentre follow-up study. Br | Psychiatry 154, 499-503

Weisman AG, Lopez SR, Ventura |, Nuechterlein KH, Goldstein MJ, Hwang S (2000) A comparison of psychiatric symptoms between Anglo-Americans and Mexican-Americans with schizophrenia. Schizophr Bull 26, 817-824

Westermeyer I (1987) Cultural factors in clinical assessment. I Consult Clin Psychol 55, 471-478

Wittkower ED, Murphy HB, Fried I, Ellenberger H (1960) Crosscultural inquiry into the symptomatology of schizophrenia. Ann N Y Acad Sci 84, 854-863

World Health Organization (1973) Report of the International Pilot Study of Schizophrenia, Vol 1. WHO Genf

World Health Organization (1979) The international pilot study of schizophrenia (IPSS). An international follow-up. Wiley Chichester

World Health Organization (1993) Klassifikation psychischer Krankheiten. Klinisch-diagnostische Leitlinien nach Kapitel V (F) der ICD-10. Huber Bern 